\title{
Assessing Use Intention and Usability of Mobile Devices in a Hybid Environment
}

Spyros Veronikis, Giannis Tsakonas and Christos Papatheodorou

Dept. of Archives and Library Sciences

Ionian University

Corfu, Greece 


\section{Presentation Layout}

- Problem statement

- Research framework description

- Building the necessary infrastructure

- Evaluation plan

- Findings 


\section{Problem definition}

- Modern information landscape includes information resources in both print and digital form.

- With the tremendous amount of available information there is a need for powerful information retrieval tools, e.g., search engines

- In hybrid environments like academic libraries physical and digital collections are physically separated and accessed from different locations, i.e., either from the stacks or from computer terminals.

- While browsing through the tangible collections one cannot access the available search tools and indexes.

- Exploration of available resources is obscured since users can not browse, search or ask an expert anytime, anywhere. 


\section{Motivation}

- The seeking strategy must allow the user to explore the tangible and digital collections iteratively.

- Remote resources such as Digital Libraries and the WWW are available anytime.

- In 2009 mobile phones were used more for data exchange rather than voice services

- Their capabilities and functionalities make them ideal to be used as information harvesting tools anytime, anywhere.

- In this work we examine and evaluate the use of mobile computing technology as a mean of unifying the seeking experience while interacting with a hybrid information environment. 


\section{Related work}

- Former applications:

- Submitt queries, exchange short messages (alerts, etc), carry around material for reference or advice (tables, images, calculators,... )

Examples: On-line Public Access Catalogs (OPACs), instant messaging, document viewers

- Latter applications:

- Mostly exploit information coming from sensors that detect location (e.g. GPS receiver) and nearby resources (e.g. RFIDtagged objects)

Examples: city guides and museum guides 


\section{Interacting with the hybrid environment}

The exploration of a hybrid information environment involves interactions between user, system and the hybrid information collections.

We extended the current system by introducing the mobile device object and studied its effect on interactions between:

a) User and collections (perceived usefulness)

b) User and mobile device (perceived usability)

c) Mobile and collections (perceived performance)

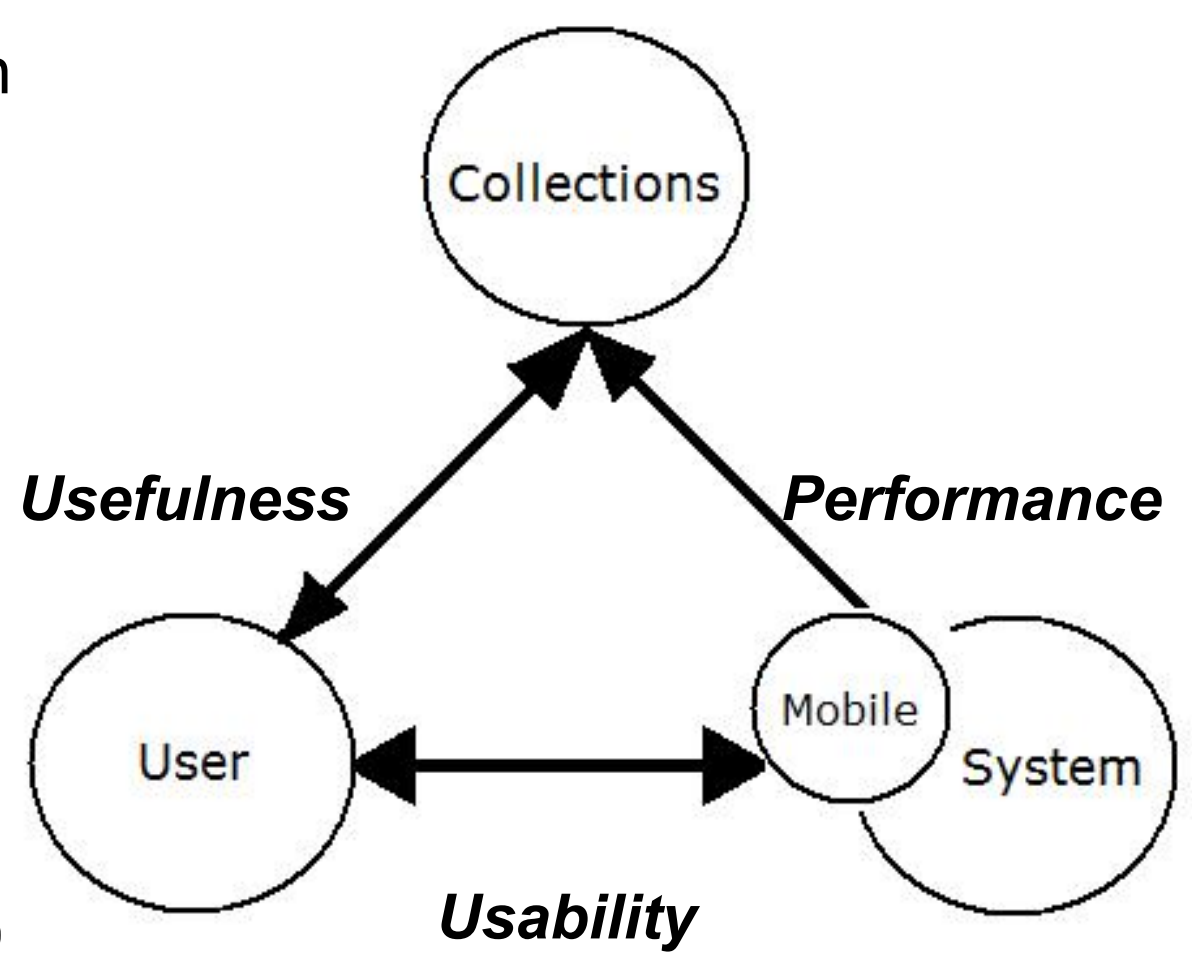




\section{Research framework}

\section{Goals:}

- Investigate the use of mobile computing devices as a mean for the seamless exploration of hybrid collections

- Gain understanding about which factors lead to the adoption of mobile services for the exploration of hybrid collections?

- Which are the most effective?

\section{Background theory}

The Unified Theory of Acceptance and Use of Technology

Use intention $=f$ (performance expectancy,

effort expectancy,

social influence,

facilitating conditions, attitude towards using tech.) 


\section{Research framework}

\section{Research hypotheses and evaluation model}

- Hypotheses are formed based on bibliography and our pilot studies.

-Hypotheses on the existence of effects are expressed as arrows.

-There are dependent and independent factors.

-Analysis will assess the existense of the effects, their size, and their statistical significance.

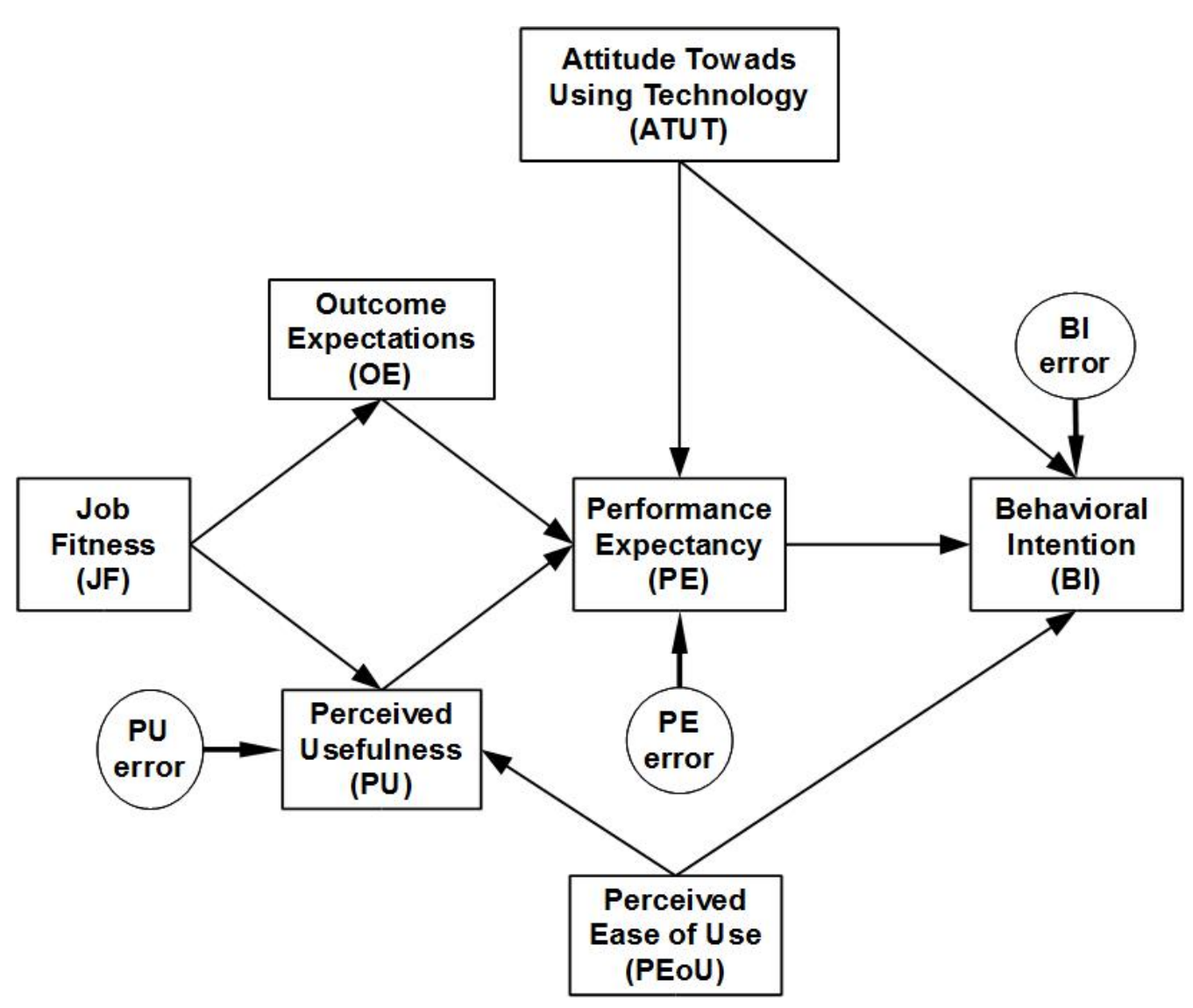




\section{Prototype description}

Main requirements

- Minimize the requirements on the mobile side

- Maintain access to existing resources
Supported functionalities

- Search tools

- Reference material

- Short message exchange

\section{Presentation}

(1)

\section{Logic}

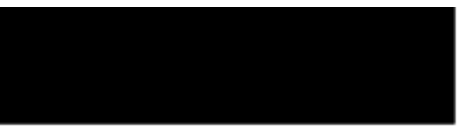

\section{Storage}

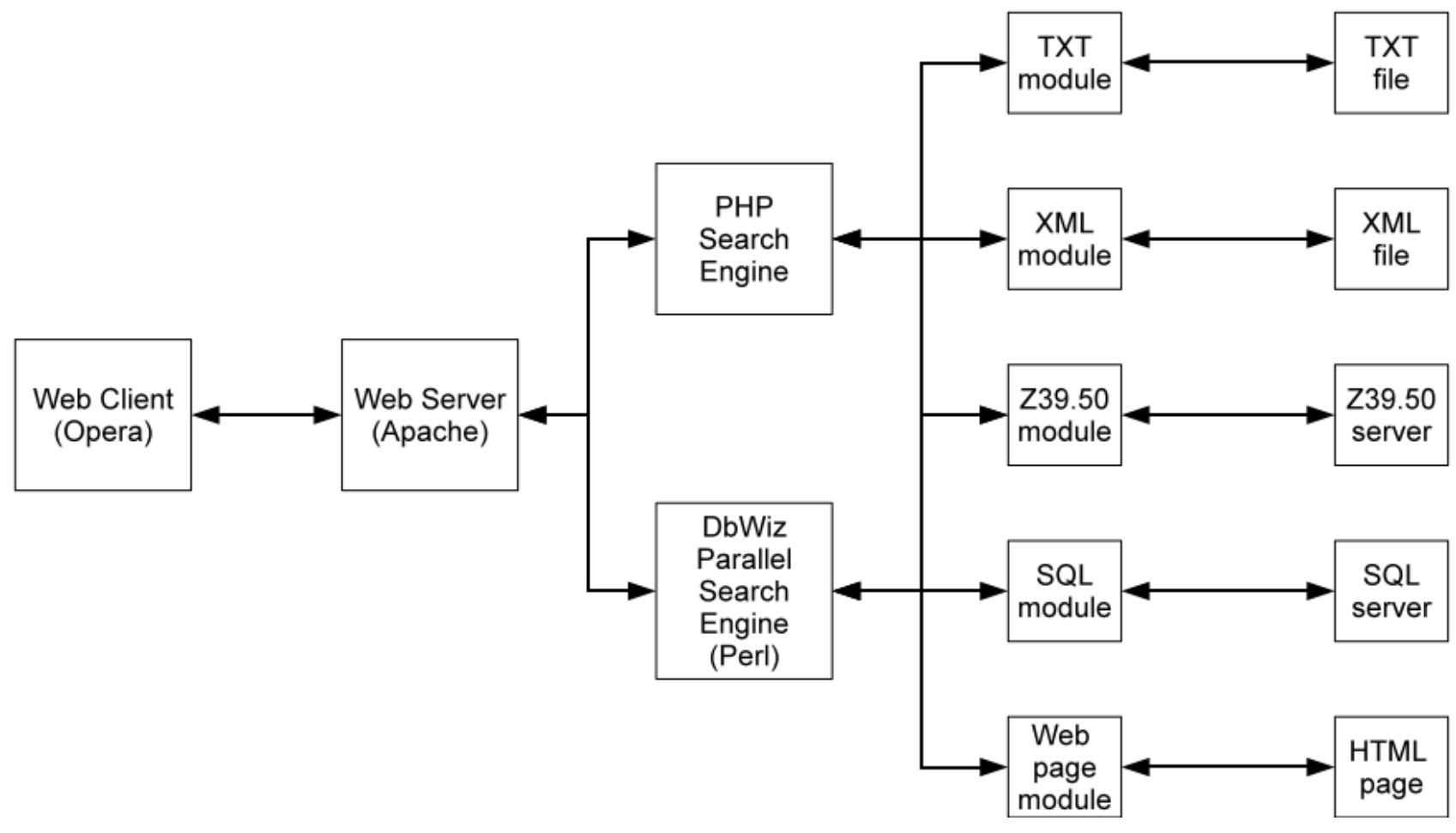

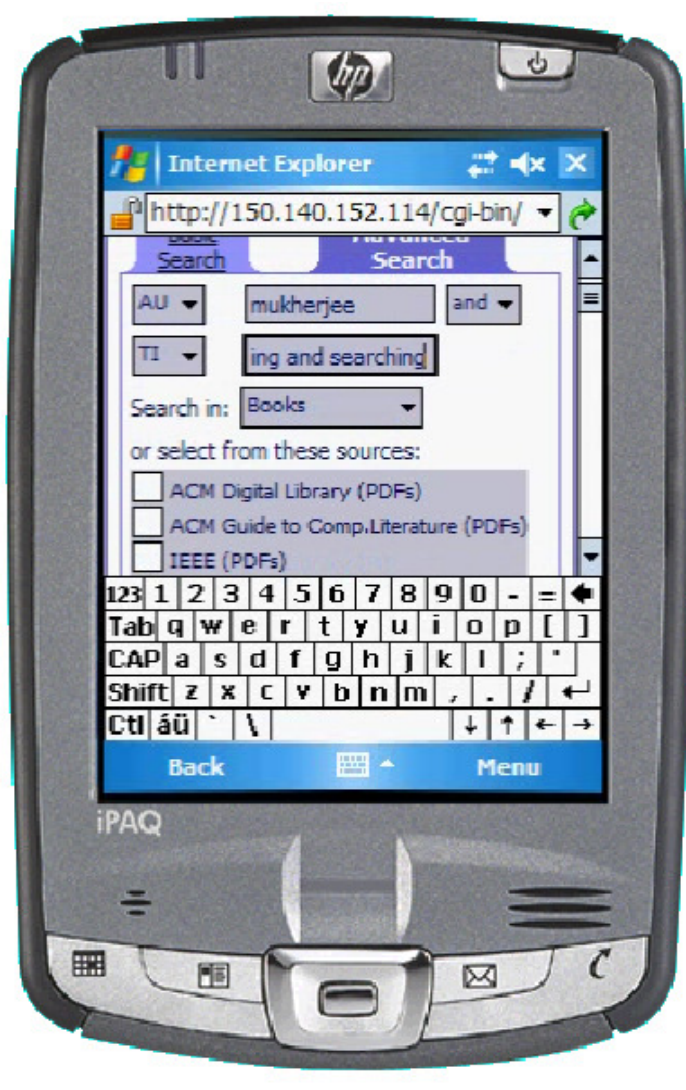




\section{Evaluation design}

- Analysis Method: path analysis and group comparisons (ANOVA)

- Data collection method: survey, where users have a hands-on experience

- Data collection tools: use intention and usability questionnaires; self administered; multi-item;

- Location: Academic library of University of Patras. It has a large collection of print and digital documents as well as access to remote digital libraries

- Participants: invited sample of library population (engineering, social and health sciences)

- Survey period: four weeks

- Collected data: 111 responses for use intention questionnaires and 83 for usability 


\section{Use intention analysis results}

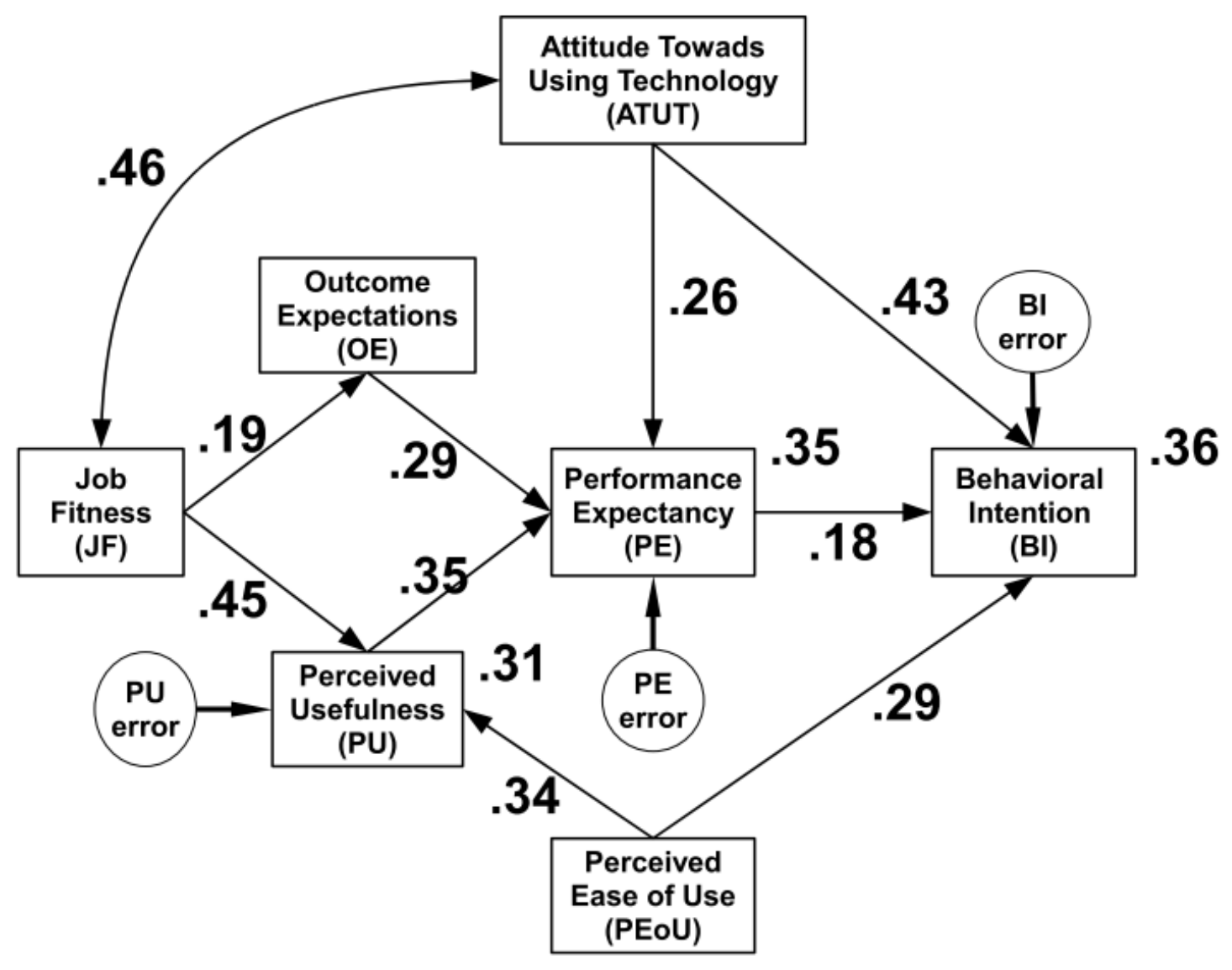

The data fit the model well, and all hypotheses were verified, and effects were found to be statistically significant.

Perceived ease of use has a direct and a mediated effect on intention to use via perceived usefulness and performance expectancy.

Attitude towards using the given technology and perceived ease of use had the strongest effect on intention to use.

Performance expectancy had a small effect.

A relation was detected between jobfitness and attitude towards using the given technology which is due to a third factor, not taken into account in our evaluation model.

In total, $36 \%$ of the recorded variance is accounted for by the model. 


\section{Use intention analysis results}

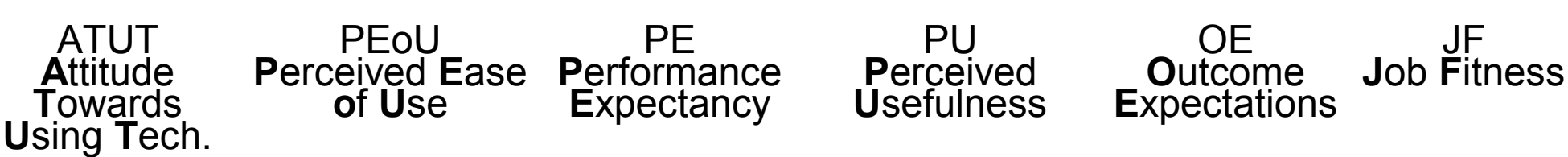

\begin{tabular}{|c|c|c|c|c|c|c|}
\hline $\begin{array}{l}\text { Perceived } \\
\text { Usefulness, PU }\end{array}$ & - & .341 & - & - & - & .446 \\
\hline $\begin{array}{l}\text { Performance } \\
\text { expectancy, PE }\end{array}$ & .257 & .120 & - & .353 & .294 & .214 \\
\hline $\begin{array}{l}\text { Behavioral } \\
\text { Intention, BI }\end{array}$ & & & .181 & .064 & .053 & .039 \\
\hline
\end{tabular}

Interpreting the values:

An increment in Perceived Ease of Use of 1 standard deviation (1.05 units) results in an increment

$$
\text { of } .309 \times 1.05=.325 \text { units in use intention score. }
$$

BI score $=4.33($ Std $=1.05), \boldsymbol{P E}>4.0$ (80\% of sample), $\boldsymbol{P U}>4.0$ (70\% of sample) 


\section{Usability analysis results}

\section{Overall usability reactions}

Overall reactions showed that users perceived the proposed approach as easy to use, adequate, effective, interesting and useful.

Usability score $=4.32($ std $=0.58)$

\section{Within-subjects comparison:}

Detect usability aspects that mostly deviate from overall usability score

Highest positive deviations were recorded on learnability items (e.g., memory load, error reduction). Lowest scores were recorded for text-input tasks (form filling, note keeping).

\begin{tabular}{lcclrc}
\hline \multicolumn{5}{c}{ Average usability score: AVG $=4.32$, STD $=0.58$} \\
\hline Overall reactions & $A V G$ & $S T D$ & Prototype characteristics & $A V G$ & $S T D$ \\
\hline Easy & 4.25 & 1.16 & Learnability & 4.67 & 0.72 \\
Satisfactory & 4.55 & 0.94 & Terms \& Language & 4.37 & 0.77 \\
Adequate & 4.63 & 0.88 & Capabilities & 4.23 & 0.73 \\
Effective & 4.62 & 0.86 & Presentation & 4.12 & 0.84 \\
Interesting & 4.70 & 1.08 & Text input & 3.72 & 1.0 \\
Useful & 4.85 & 0.88 & & & \\
\hline
\end{tabular}

\begin{tabular}{lcc}
\hline Top positively deviated items & t-value STD \\
\hline 1. Learnability: error reduction & 12.08 & 0.95 \\
2. Learnability: memory load & 7.62 & 0.63 \\
3. Effectiveness: search results' relevance & 7.07 & 0.58 \\
4. Useful: usefulness & 5.90 & 0.52 \\
5. Interest: service perceived as interesting & 4.13 & 0.58 \\
\hline Top negatively deviated items & & \\
\hline 1. Text input: typing minimization & -7.76 & -1.22 \\
2. Presentation: screen navigation & -6.44 & -0.85 \\
3. Text input: typing speed & -4.60 & -0.53 \\
4. Text input: self error correction & -3.35 & -0.38 \\
5. Capabilities: response time & -3.20 & -0.34 \\
\hline
\end{tabular}

\section{Between-subjects comparison:}

Test for differences in usability according to experience level (Sci./Eng. vs Soc./Health sciences) Test of usability difference between two groups was found to be non significant, i.e., previous experience does not affect usability

ANOVA for usability accross 4 levels of experience (none, some, medium, high) revealed no significant differences. 


\section{Conclusion}

Users were satisfied with the user-centric approach for the exploration of the hybrid environment and are willing to try new tools and services.

We found out that attitude towards using the mobile technology, as well as performance and effort expectancy have a significant effect on intention to use it; the former had the strongest effect.

Ease of use positively affects intention to use both directly and via perceived usefulness and performance expectancy, therefore it is important to improve usability.

Learning to use the given technology was easy because its based on known tasks and methods, just extended on a mobile platform.

The usability analysis showed that users perceive the mobile technology as easy to use, regardless of their level of experience with it, making it usable by a wide group of users.

The number of mobile computing devices such as smartphones is constantly increasing, thus becoming ubiquitous and making information delivery available anytime, anywhere. Our study recorded a clear attitude of users towards using it as a means of unifying their seeking experience, while they maintain an active role in the exploration of the available information resources. 


\section{Acknowledgements}

This work was funded by the PENED'03/791 research project, which is co-financed by the E.U. - European Union Social Fund (80\%) and the Greek Ministry of Development, GSRT (20\%).

Thank you for your attention. 